\title{
Complete Characterization of a Quantum Process: the Two-Bit Quantum Gate
}

\author{
J. F. Poyatos, J. I. Cirac \\ Departamento de Fisica Aplicada, Universidad de Castilla-La Mancha, 13071 Ciudad Real, Spain \\ P. Zoller \\ Institut für Theoretische Physik, Universität Innsbruck, A-6020 Innsbruck, Austria
}

(February 9, 2008)

\begin{abstract}
We show how to fully characterize a quantum process in an open quantum system. We particularize the procedure to the case of a universal two-qubit gate in a quantum computer. We illustrate the method with a numerical simulation of a quantum gate in the ion trap quantum computer.

PACS Nos. 03.65.Bz, 42.50.Lc, 42.50.Wm
\end{abstract}

Recently there has been a growing interest in "quantum tomography", i.e. in the complete characterization of the state of a quantum system represented by a density operator $\hat{\rho}$. Quantum tomography of an unknown quantum state (that can be repeatedly prepared) [1] consists of finding an appropriate sequence of measurements which allows one to determine the complete density operator $\hat{\rho}$ (for experimental implementations and theoretical schemes in quantum optics see [1]). In this letter we will show how to completely characterize a physical process in an open quantum system. More specifically, suppose that a given quantum dynamics $\mathcal{E}$ transforms input states $\rho_{\text {in }}$ into output states $\rho_{\text {out }}$, i.e.

$$
\hat{\rho}_{\text {in }} \stackrel{\mathcal{E}}{\longrightarrow} \hat{\rho}_{\text {out }}=\mathcal{E}\left[\hat{\rho}_{\text {in }}\right]
$$

with $\mathcal{E}$ a linear mapping. Our aim is to characterize the process $\mathcal{E}$, given as a "black box", by a sequence of measurements in such a way that it is possible to predict what the output state will be for any input state.

The particular problem that we will analyze after developing a general formalism is the characterization of the two-bit universal quantum gate for quantum computing [2]. A quantum computer consists of $n$ two-level atoms with atomic states $|0\rangle_{i},|1\rangle_{i}(i=1, \ldots, n)$ representing the quantum bits (qubits). States of the quantum computer are $n$-atom entangled states in the product Hilbert space $|\psi\rangle \in \mathcal{H}=\prod_{i} \otimes \mathcal{H}_{2}(i)$ with $\mathcal{H}_{2}(i)=\left\{|0\rangle_{i},|1\rangle_{i}\right\}$. Quantum computations correspond to physical processes $\left|\psi_{\text {out }}\right\rangle=\hat{U}\left|\psi_{\text {in }}\right\rangle$ where a given input state is mapped to an output state by a unitary transformation $\hat{U}$. This can be carried out as a sequence of elementary steps (quantum gates) involving operations on a few qubits. It has been shown that any computation can be decomposed into single-bit gates, and a universal two-bit gate which involves an entanglement operation on two qubits [2]. In reality, due to the presence of decoherence and experimental imperfections, these gates (and therefore any computation) will not be ideal. In present experiments related to quantum computing based on both laser cooled trapped ions [3] and atoms in cavities [4], the difficult part is the two-qubit gate, since it requires an interaction between the two two-level systems via an auxiliary system (phonons or photons) which leads to decoherence. In view of this fact, we wish to develop a procedure for characterizing a two-qubit gate, i.e. characterize a physical process $\mathcal{E}$ involving entanglement of two qubits 1 and 2 in the state space $\mathcal{H}_{2}(1) \otimes \mathcal{H}_{2}(2)$. Below we will show how to implement this using only product states as inputs, and single qubit measurements on the outputs (assuming that single bit preparations and operations can be performed reliably ). We avoid utilizing any interaction (entanglement) between the qubits which would be required to prepare Bell state inputs and perform Bell measurements since otherwise the decoherence and errors induced by the measurement itself would distort the characterization of $\mathcal{E}$. Furthermore, we will introduce four global parameters to characterize the action of the quantum gate: the "Gate Fidelity" $(\mathcal{F})$, the "Gate $\mathrm{Pu}-$ rity" $(\mathcal{P})$, the "Quantum Degree" $(\mathcal{Q})$, and the "Entanglement Capability" $(\mathcal{E})$. These four parameters can be calculated once the physical process is completely determined. To illustrate the procedure of measuring $\mathcal{E}$, we will analyze below simulation data from a model of a two-bit quantum gate in an ion trap quantum computer [5.

To develop the general formalism, let us consider an experiment in which a quantum system undergoes a physical process $\mathcal{E}$. We assume that the system is initially prepared in the pure state

$$
\left|\Psi_{\text {in }}\right\rangle=\sum_{i=0}^{N} c_{i}|i\rangle \in \mathcal{H}_{\mathrm{in}},
$$

where $|0\rangle,|1\rangle, \ldots,|N\rangle$ are orthogonal states spanning the Hilbert space of (allowed) input states $\mathcal{H}_{\text {in }}$ with dimension $N+1$, a subspace of the system Hilbert space $\mathcal{H}_{\mathrm{S}}$. We will denote by $\left.\rho_{E}=\sum_{\alpha} \omega_{\alpha} \mid E^{\alpha}\right)\left(E^{\alpha} \mid\right.$ the initial state of the environment, i.e., of the other degrees of freedom that will be coupled to our system. In its most general form, the physical process will perform a transformation defined by 


$$
\left.\left.|i\rangle \mid E^{\alpha}\right) \stackrel{\mathcal{E}}{\longrightarrow} \sum_{j=0}^{M}|j\rangle \mid E_{i j}^{\alpha}\right), \quad(i=0, \ldots, N)
$$

where in the sum we have taken into account the possible presence of other system states that might be populated during the interaction (i.e., $M \geq N$ in general; see also below for the case of a quantum gate). The states $\left.\mid E_{i j}^{\alpha}\right)$ are unnormalized states of the environment. Combining (2) and (3), and tracing over the environment degrees of freedom we get the following reduced system density operator,

$$
\hat{\rho}_{\text {out }}=\sum_{i, i^{\prime}=0}^{N} c_{i}\left[c_{i^{\prime}}\right]^{*} \hat{R}_{i^{\prime} i}
$$

in the space of output states, $\mathcal{H}_{\text {out }}=\{|0\rangle, \ldots,|M\rangle\}$, and where

$$
\hat{R}_{i^{\prime} i} \equiv \sum_{j, j^{\prime}=0}^{M}|j\rangle\left\langle j^{\prime}\right| \sum_{\alpha} \omega_{\alpha}\left(E_{i^{\prime} j^{\prime}}^{\alpha} \mid E_{i j}^{\alpha}\right) \quad\left(i, i^{\prime}=0, \ldots, N\right)
$$

are system operators that do not depend on the initial state. From the knowledge of these operators one can predict the final density operator $\hat{\rho}_{\text {out }}$ for any input state $\rho_{\text {in }}$. For a mixed initial state, we diagonalize $\hat{\rho}_{\text {in }}=$ $\sum_{n} p_{n}\left|\Psi_{\text {in }}^{(n)}\right\rangle\left\langle\Psi_{\text {in }}^{(n)}\right|$ and use the fact that the evolution $\mathcal{E}$ is linear, which leads to $\hat{\rho}_{\text {out }}=\sum_{n} p_{n} \mathcal{E}\left(\left|\Psi_{\text {in }}^{(n)}\right\rangle\left\langle\Psi_{\text {in }}^{(n)}\right|\right)$. Thus, the problem of fully characterizing the physical process $\mathcal{E}$ on the system is reduced to finding the $(N+1)^{2}$ "transfer operators" $\hat{R}_{i^{\prime} i}$ in $\mathcal{H}_{\text {out }}$. They fulfill $\operatorname{Tr}\left\{\hat{R}_{i^{\prime} i}\right\}=\delta_{i^{\prime} i}$ and $\left(\hat{R}_{i^{\prime} i}\right)^{\dagger}=\hat{R}_{i i^{\prime}}$.

To develop a procedure to measure these operators we define two vectorial operators

$$
\begin{aligned}
\vec{\rho}_{\text {out }} & \equiv\left\{\hat{\rho}_{\text {out }}^{(1)}, \hat{\rho}_{\text {out }}^{(2)}, \ldots, \hat{\rho}_{\text {out }}^{(N+1)^{2}}\right\}, \\
\vec{R} & \equiv\left\{\hat{R}^{1}, \hat{R}^{2}, \ldots, \hat{R}^{(N+1)^{2}}\right\}
\end{aligned}
$$

Here, the components of $\vec{\rho}_{\text {out }}$ are a set of output operators corresponding to $(N+1)^{2}$ different initial inputs of the form (2) with coefficients $c_{i}^{(k)}\left[k=1, \ldots,(N+1)^{2}\right]$. All these density operators can be fully characterized using standard quantum tomography methods [1]. On the other hand, the components of the vector $\vec{R}$, are defined according to $\hat{R}_{q}=\hat{R}_{i^{\prime} i}$, with $q=(N+1) i^{\prime}+i+1$. In view of (4), these two vectorial operators are related by a set of linear equations, written in a matrix form as $\vec{\rho}_{\text {out }}=\mathcal{M} \vec{R}$, where $\mathcal{M}$ is a (c-number) matrix whose elements are defined as $\mathcal{M}_{k q}=c_{i}^{(k)}\left[c_{i^{\prime}}^{(k)}\right]^{*}$. The problem of obtaining the transfer operators $\vec{R}$ thus reduces to finding a set of initial states of the system, such that the matrix $\mathcal{M}$ is not singular. In that case we will have $\vec{R}=\mathcal{M}^{-1} \vec{\rho}_{\text {out }}$, which solves the problem. Next, we prove that such an invertible matrix $\mathcal{M}$ exists by explicit construction. One can simply choose the initial states as follows:

$$
c_{i}^{(k)}= \begin{cases}\frac{1}{\sqrt{2}}\left(\delta_{i k_{1}}+\delta_{i k_{2}}\right) & \text { if } k_{1}>k_{2}, \\ \delta_{i k_{1}} & \text { if } k_{1}=k_{2}, \\ \frac{1}{\sqrt{2}}\left(\delta_{i k_{1}}+i \delta_{i k_{2}}\right) & \text { if } k_{1}<k_{2},\end{cases}
$$

with $k=(N+1) k_{1}+k_{2}$.

Let us now apply the above procedure to a universal two-qubit gate. Now, the system is composed of two two-level subsystems 1 and 2 of levels $|0\rangle_{1,2}$ and $|1\rangle_{1,2}$ each. We can define a set of orthogonal states $|i\rangle=$ $\left|i_{1}\right\rangle_{1}\left|i_{2}\right\rangle_{1}$ (with $i=2 i_{1}+i_{2}$, and $i_{1}, i_{2}=0,1$ ) and write the initial states as in (2) (with dimension $N+1=4$ ). The quantum tomography of the output states can be carried out following the lines proposed by Wootters [6]: one writes the output density operator as

$$
\hat{\rho}_{\text {out }}=\sum_{q=0}^{15} \lambda_{q} \hat{A}_{q},
$$

where $\hat{A}_{q}=\hat{\sigma}_{q_{1}}^{1} \otimes \hat{\sigma}_{q_{2}}^{2}\left(q=4 q_{1}+q_{2}\right)$, with $\hat{\sigma}_{q_{i}}^{a}=$ $\left\{\hat{1}^{a}, \hat{\sigma}_{x}^{a}, \hat{\sigma}_{y}^{a}, \hat{\sigma}_{z}^{a}\right\}$ and $a=1,2$ refers to the first and second qubit, respectively. By measuring the observables $\hat{A}_{q}$, one can determine the coefficients $\lambda_{q}$, given that $\lambda_{q}=\operatorname{Tr}\left[\hat{\rho}_{\text {out }} \hat{A}_{q}\right] / 4$ [7]. Note that all these measurements do not require any interaction between the qubits (Bell state measurements), that is, for all these measurements the two qubits can be measured independently, without the application of another two-qubit gate. This is needed since otherwise the measurement procedure would involve errors that could not be separated from the gate itself. However, some of the 16 initial states given above in order to make up the matrix $\mathcal{M}$ [c.f. Eq. (7)] are entangled states. Their preparation would involve the application of a two-qubit gate which would also lead to uncontrollable errors. Fortunately, there are other sets of initial states that are unentangled for which the matrix $\mathcal{M}$ is invertible. An example are the 16 product states $\left|\psi_{a}\right\rangle_{1}\left|\psi_{b}\right\rangle_{2}(a, b=1, \ldots, 4)$, where

$$
\begin{aligned}
\left|\psi_{1}\right\rangle & =|0\rangle, \quad\left|\psi_{3}\right\rangle=\frac{1}{\sqrt{2}}(|0\rangle+|1\rangle) \\
\left|\psi_{2}\right\rangle & =|1\rangle, \quad\left|\psi_{4}\right\rangle=\frac{1}{\sqrt{2}}(|0\rangle+i|1\rangle) .
\end{aligned}
$$

In order to illustrate this procedure, we have studied a two-qubit gate in the ion trap quantum computer model [5]. We have considered two ions in a linear ion trap interacting with two lasers. Let us denote by $|g\rangle_{n} \equiv|0\rangle_{n}$ and $|e\rangle_{n} \equiv|1\rangle_{n}$ two internal states of the $\mathrm{n}$-th ion, and by $\left|e^{\prime}\right\rangle_{n}$ an auxiliary internal state. As we have shown in Ref. [5], the universal two-qubit gate defined by

$$
\left|\epsilon_{1}\right\rangle_{1}\left|\epsilon_{2}\right\rangle_{2} \rightarrow(-1)^{\epsilon_{1} \epsilon_{2}}\left|\epsilon_{1}\right\rangle_{1}\left|\epsilon_{2}\right\rangle_{2}, \quad\left(\epsilon_{1,2}=0,1\right)
$$

can be implemented in three steps: (i) Apply a $\pi$ laser pulse to the lower motional sideband corresponding to the transition $|g\rangle_{1} \rightarrow|e\rangle_{1}$ of the first ion; (ii) Apply a $2 \pi$ 
laser pulse to the lower motional sideband of the transition $|g\rangle_{2} \rightarrow\left|e^{\prime}\right\rangle_{2}$ of the second ion; (iii) as (i). By lower motional sideband we mean that the laser frequency has to be equal to the corresponding internal transition frequency minus the trap frequency, in order to excite a center of mass phonon only. The interaction of the two ions and the laser is given by the following Hamiltonian 8

$$
\begin{aligned}
H= & -\Delta_{1}|e\rangle_{11}\left\langle e\left|-\Delta_{2}\right| e^{\prime}\right\rangle_{22}\left\langle e^{\prime}\right|+\nu a_{\mathrm{cm}}^{\dagger} a_{\mathrm{cm}}+\sqrt{3} \nu a_{\mathrm{r}}^{\dagger} a_{\mathrm{r}} \\
& +\frac{\Omega_{1}(t)}{2}\left[|e\rangle_{11}\langle g| e^{-i \eta_{\mathrm{cm}}\left(a_{\mathrm{cm}}+a_{\mathrm{cm}}^{\dagger}\right)} e^{-i \eta_{\mathrm{r}}\left(a_{\mathrm{r}}+a_{\mathrm{r}}^{\dagger}\right)}+\text { H.c. }\right] \\
& +\frac{\Omega_{2}(t)}{2}\left[\left|e^{\prime}\right\rangle_{22}\langle g| e^{-i \eta_{\mathrm{cm}}\left(a_{\mathrm{cm}}+a_{\mathrm{cm}}^{\dagger}\right)} e^{i \eta_{\mathrm{r}}\left(a_{\mathrm{r}}+a_{\mathrm{r}}^{\dagger}\right)}+\text { H.c. }\right]
\end{aligned}
$$

Here, $\Delta_{1,2}$ and $\Omega_{1,2}$ are the laser detunings and Rabi frequencies of the laser acting on each ion, respectively. The operators $a$ and $a^{\dagger}$ are annihilation and creation operators of the center of mass $(\mathrm{cm})$ and relative $(\mathrm{r}) \mathrm{mo}-$ tion mode, $\eta$ is the corresponding Lamb-Dicke parameter, and $\nu$ is the trap frequency.

We have calculated numerically the evolution given by this Hamiltonian. Following the steps mentioned above, and for Rabi frequencies much smaller than the trap frequency this evolution corresponds to the gate (10] [5]. For finite Rabi frequencies, however, the result will not be ideal. After the gate operation, there will remain some population in the phonon modes, which would lead to decoherence. Moreover, there may remain some population in the auxiliary state $\left|e^{\prime}\right\rangle_{2}$, and therefore in this case we have $M=4>N=3$. With the numerical calculation we have simulated the measurement of the operators $\hat{R}_{i^{\prime} i}$, that fully characterize the evolution process. We emphasize that all the information regarding this (non-ideal) gate is contained in these operators. In Fig. 1 we have compared the ideal case of a perfect gate [Fig. 1(a)] with the simulation results with realistic parameters [Figs. $1(\mathrm{~b}-\mathrm{d})]$. We have plotted the matrix elements of 16 operators $\hat{R}_{i^{\prime} i}$ sorted according to $\mathcal{E}_{n, m}=\left\langle j^{\prime}\left|\hat{R}_{i^{\prime} i}\right| j\right\rangle$, with $n=4 i+j$ and $m=4 i^{\prime}+j^{\prime}$. We have chosen a set of parameters close to those planned in experiments: $\eta_{\mathrm{cm}}=\eta_{\mathrm{r}}=0.5, \Delta_{1}=\Delta_{2}=-\nu$, and Rabi frequencies $\Omega_{1}=\Omega_{2}=0.1,0.2 \nu$ and 0.5 [Figs.1(b,c,d)] [9]. As it is shown, for moderately small Rabi frequencies the simulated results almost coincide with the ideal ones.

In order to elucidate to what extent a two-qubit gate implemented experimentally in a particular model of quantum computer approaches to the ideal one, we define a parameter, the "Gate Fidelity", as

$$
\mathcal{F}=\overline{\left\langle\Psi_{\text {in }}\left|\hat{U}^{\dagger} \hat{\rho}_{\text {out }} \hat{U}\right| \Psi_{\text {in }}\right\rangle}
$$

where the overline indicates average over all possible input states $\left|\Psi_{\text {in }}\right\rangle$, and $\hat{U}$ is the unitary operator corresponding to the ideal gate. This parameter can be calculated once the full characterization of the gate is performed using

$$
\mathcal{F}=\frac{1}{8} \sum_{i=0}^{3} F_{i i}^{i i}+\frac{1}{24} \sum_{i \neq j}\left(F_{j j}^{i i}+F_{i j}^{j i}\right),
$$

where $F_{j^{\prime} j}^{i^{\prime} i} \equiv\left\langle j^{\prime}\left|\hat{U}^{\dagger} \hat{R}_{i^{\prime} i} \hat{U}\right| j\right\rangle$. Obviously, a gate fidelity close to one indicates that the gate was carried out almost ideally.

In a similar way we can define the "Gate Purity" $\mathcal{P}=\overline{\operatorname{Tr}\left\{\left(\hat{\rho}_{\text {out }}\right)^{2}\right\}}$ that reflects the effects of decoherence on the gate. $\mathcal{P}$ close to one indicates that the effects of decoherence are negligible. It can be shown that

$$
\mathcal{P}=\frac{1}{8} \sum_{i=0}^{3} \operatorname{Tr}\left\{\left(\hat{R}_{i i}\right)^{2}\right\}+\frac{1}{24} \sum_{i \neq j} \operatorname{Tr}\left\{\hat{R}_{i i} \hat{R}_{i^{\prime} i^{\prime}}+\hat{R}_{i^{\prime} i} \hat{R}_{i i^{\prime}}\right\}
$$

In addition, the "Quantum Degree of the Gate" $\mathcal{Q}$ is defined as the maximum value of the overlap between all possible output states that are obtained starting from an unentangled state and all the maximally entangled states, i.e.

$$
\mathcal{Q}=\max _{\tilde{\rho}_{\text {out }},\left|\Psi_{\text {me }}\right\rangle}\left\langle\Psi_{\text {me }}\left|\tilde{\rho}_{\text {out }}\right| \Psi_{\text {me }}\right\rangle
$$

where $\tilde{\rho}_{\text {out }}$ denote the output states corresponding to unentangled input states $\left|\Psi_{\text {in }}\right\rangle=\left|\psi_{a}\right\rangle_{1}\left|\psi_{b}\right\rangle_{2}$, and $\left|\Psi_{\text {me }}\right\rangle$ is a maximum entangled state [10]. As it has been shown, when the overlap between a density operator and a maximally entangled state is larger than $(2+3 \sqrt{2}) / 8 \simeq 0.78$, Clauser-Horne-Shimony-Holt (CHSH) inequalities are violated [11. Finally, another useful parameter is the "Entanglement Capability" $\mathcal{E}[10$, given as the smallest eigenvalue of the partial transposed density matrix $\hat{\rho}_{\text {out }}$, for unentangled inputs states. As it has been recently shown [10], the negativity of this quantity is a necessary and sufficient condition for non-separability of density operators of two spin-1/2 systems. These quantities can be calculated numerically starting from the gate operators $\hat{R}_{i^{\prime} i}$ with a maximization/minimization procedure.

In Fig. 2 we have plotted these four parameters as a function of the Rabi frequency $\Omega \equiv \Omega_{1}=\Omega_{2}$ for three different Lamb-Dicke parameters and the same parameters as in Fig. 1. As expected, the best results are obtained for small Rabi frequencies. This is due to the fact that transitions to undesired levels are suppressed. On the other hand, a Lamb-Dicke parameter close to one also improves the results. This is due to the fact that in contrast to Ref. [5] we are considering here traveling wave excitation, in which transitions that do not change the phonon number can be excited by the laser. The ratio between the effective Rabi frequencies of the lower sideband and these unwanted ones is proportional to $\eta$ in the Lamb-Dicke limit. The figures also indicate that the $\mathcal{F}$, $\mathcal{P}, \mathcal{Q}$, and $\mathcal{E}$ decay in a different manner when the parameters deviate from the conditions of operation of the ion trap quantum computer.

Finally, we wish to mention another application of this procedure of characterization of a quantum physical process. Let us consider a quantum system coupled to a 
Markovian reservoir. In the case that the Hamiltonian is time independent, one can describe the evolution of the reduced system density operator $\hat{\rho}$ in terms of a master equation of the form $\dot{\hat{\rho}}=\mathcal{L} \hat{\rho}$, where $\mathcal{L}$ is a Liouvillian superoperator. The formal solution to this equation after a time $t$ is $\hat{\rho}_{\text {out }}=e^{\mathcal{L} t} \hat{\rho}_{\text {in }}$. The full characterization of the physical process in this case would allow one to "measure" the Liouvillian $\mathcal{L}$, and therefore to determine the master equation fulfilled by the system. Moreover, choosing different interaction times one could check whether a given process is Markovian or not.

In conclusion, we have shown how to perform the full characterization of a physical process. This requires the preparation of various initial states, and the quantum tomography of the corresponding output states. We have illustrated these results for the case of a quantum gate, and have defined four parameters that give the quality of the gate. In addition, we have presented numerical simulations for the ion trap quantum computer. The complete characterization of the quantum gates is relevant both from an experimental point of view to evaluate implementations of quantum gates in the laboratory, as well as a theoretical tool to compare the expected performance of specific quantum computer model systems.

We thank A. Barenco, J. Kimble, H. Mabuchi, T. Pellizzari, and R. Walser for discussions. This work was supported by the Austrian Science Foundation, "Acciones Integradas", and the European TMR network ERB4061PL95-1412. J. F. Poyatos acknowledges the "J. C. Castilla-La Mancha".

[1] For a review see, for example, U. Leonhardt and H. Paul, Prog. Quantum Electron. 19, 89 (1995), and references cited.

[2] See for example, D. P. DiVincenzo, Science 270, 255 (1995), and A. Ekert and R. Jozsa, Rev. Mod. Phys. (to be published) and references therein.

[3] C. Monroe et al., Phys. Rev. Lett. 75, 4714 (1995).

[4] Q. A. Turchette et al., Phys. Rev. Lett. 75, 4710 (1995); M. Brune et al., ibid. 76, 1800 (1996).

[5] J. I. Cirac and P. Zoller, Phys. Rev. Lett. 74, 4091 (1995).

[6] W.K. Wooters, Ann. Phys. (N.Y.) 176, 1 (1987).

[7] In some gate models auxiliary internal atomic levels are used, and the output states can have amplitudes in those levels $(M>N=3)$. In Eq. (8), $\hat{\rho}_{\text {out }}$ denotes a projection of the density operator on $\mathcal{H}_{2}(1) \otimes \mathcal{H}_{2}(2)$, i.e. $\operatorname{Tr} \hat{\rho}_{\text {out }}<1$ which is the probability of ending up in the right Hilbert space.

[8] In contrast to Ref. [5] we have chosen lasers in a traveling wave configuration.

[9] In Ref. 5], the Lamb-Dicke limit $\eta_{\mathrm{cm}} \ll 1$ was assumed. One can extend the results beyond the Lamb-Dicke limit by correcting the time duration of the laser pulses. Here we have used these corrected times.
[10] A. Peres, Phys. Rev. Lett. 77, 1413 (1996); R. Horodecki, P. Horodecki, and R. Horodecki, Report No. quantnh /960.5ก38
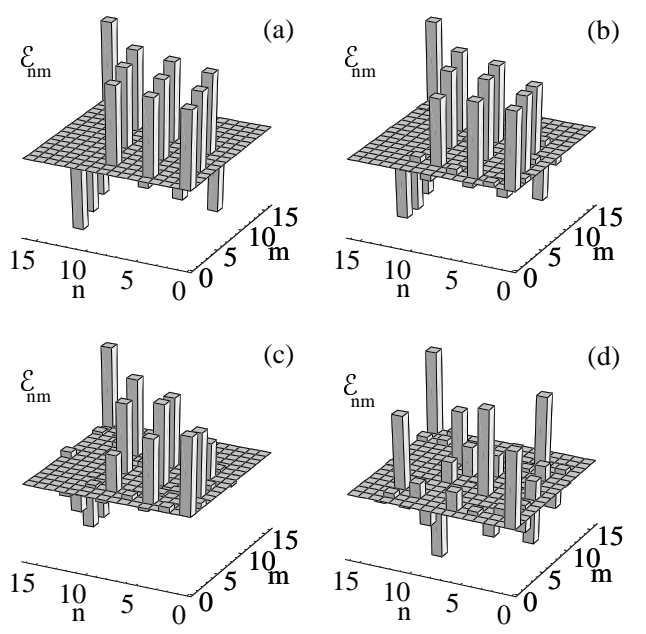

FIG. 1. $\mathcal{E}_{n, m}$. in arbitrary units (see text for explanation) for a two-qubit gate in the ion trap quantum computer: (a) ideal gate; (b,c,d) numerical simulation with the following parameters: $\eta_{\mathrm{cm}}=\eta_{\mathrm{r}}=0.5, \Delta_{1}=\Delta_{2}=-\nu$, and $\Omega_{1}=\Omega_{2}=0.1,0.2,0.5 \nu$ $(b, c, d)$
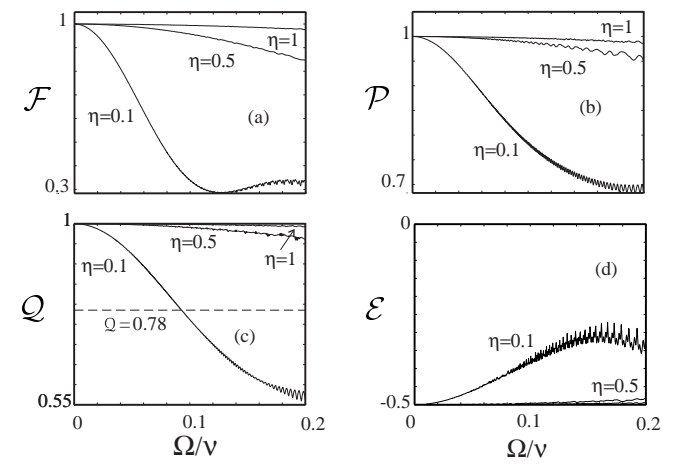

FIG. 2. Fidelity (a), Purity (b), Quantum Degree (c) and Entanglement Capability (d) for a two-qubit gate in the ion trap quantum computer as a function of the Rabi frequency. The values of the Lamb-Dicke parameter are indicated. All the other parameters are as in Fig. 1 